\title{
Die Anderen als sozialer Kontext. Zur Bedeutung strategischer Interaktion
}

\section{Journal Article}

\section{Author(s):}

Diekmann, Andreas

Publication date:

2014-09

\section{Permanent link:}

https://doi.org/10.3929/ethz-b-000097268

\section{Rights / license:}

In Copyright - Non-Commercial Use Permitted

\section{Originally published in:}

Kölner Zeitschrift für Soziologie und Sozialpsychologie 66, https://doi.org/10.1007/s11577-014-0258-5 


\title{
Die Anderen als sozialer Kontext. Zur Bedeutung strategischer Interaktion
}

\author{
Andreas Diekmann
}

(C) Springer Fachmedien Wiesbaden 2014

Zusammenfassung Strategisches Handeln bedeutet, dass die Ergebnisse einer Handlung von „Ego“ von den Handlungen der „Alteri“ abhängig sind. Solche Situationen sind zahlreich im Alltagsleben, in Wirtschaft und Politik. Pioniere wie Erving Goffman oder Raymond Boudon haben die Bedeutung strategischen Handelns für die soziologische Analyse frühzeitig erkannt. Die Handlungsmöglichkeiten anderer Personen bilden den strategischen Kontext einer Person. Zwischen Kontext und Handlung besteht eine Wechselwirkung, deren Dynamik mit spieltheoretischen Modellen analysiert werden kann. Anhand der drei Beispiele Verantwortungsdiffusion, Wettbewerb und relative Deprivation, sozialer Tausch und Vertrauensproblem wird strategisches Handeln erläutert und aufgezeigt, dass strategischer Kontext die Ressourcen und die Wahrnehmung von Chancen beeinflusst, die in Analysen strategischen Handelns eben nicht als exogen angenommen werden können. Analysen des strategischen Kontexts bewegen sich sowohl auf der Mikro- als auch auf der Makroebene. Allerdings sind die strikten Rationalitätsanforderungen der Modelle nicht immer erfüllt. Oft sind evolutionäre Erklärungen angemessener, die auf weniger restriktiven Annahmen adaptiven Verhaltens basieren.

Schlüsselwörter Strategisches Handeln · Strategische Kontexte · Verantwortungsdiffusion · Relative Deprivation

\footnotetext{
A. Diekmann $(\bowtie)$

CLU D 3, Eidgenössische Technische Hochschule Zürich,

Clausiusstraße 50, 8092 Zürich, Schweiz

E-Mail: diekmann@soz.gess.ethz.ch
} 


\section{Actor's Social Context. The Importance of Strategic Interaction}

Abstract An action is defined as "strategic" when the consequences of ego's action depend on the action of alter. Situations of strategic interaction are numerous in daily life, business, and politics. Pioneers like Erving Goffman or Raymond Boudon recognized the importance of strategic interaction in sociological analysis long ago. Other peoples' opportunities of actions form ego's strategic context. The dynamics of the impact of the strategic context on ego's action can be modeled and analyzed by means of game theory. We will discuss three examples of strategic interaction models: "Diffusion of responsibility", Boudon's "logic of relative frustration", and the problem of social exchange and trust. We demonstrate the effects of the strategic context on the opportunities and beliefs of actors. In contrast to non-strategic rational choice theory, beliefs and opportunities are not assumed as exogenous. The analysis of the strategic context contributes to a better understanding of the micro-level effects and the macro-level implications. However, the strict rationality requirements of game models are often violated. In these situations, evolutionary models based on principles of learning and adaptions are more adequate than models based on assumptions of strict rationality.

Keywords Strategic contexts - Strategic interaction - Diffusion of responsibility · Relative deprivation

\section{Einleitung: Kontext und soziales Handeln}

Ein Patient sucht seinen Arzt nach einem positiven Testergebnis einer Vorbeugeuntersuchung auf. Der Arzt rät zu einer speziellen Therapie. Es besteht das Risiko, dass der Arzt zwar nach bestem Wissen und Gewissen handelt, jedoch nicht auf dem neuesten Stand seiner Wissenschaft ist und eine wenig erfolgreiche Therapie verschreibt. Ein weiteres Risiko besteht darin, dass der Arzt zwar bestens informiert ist, aber nicht die optimale Therapie verschreibt, weil er selbst von einer bestimmten Behandlungsmethode profitiert. Der Patient hat also, anders als ein Flugpassagier, der der Kompetenz des Piloten vertraut, ein doppeltes Vertrauensproblem (siehe Gigerenzer 2013). Ähnlich geht es dem Kunden einer Autoreparaturwerkstatt, dem Kunden eines Anlageberaters einer Bank oder dem Klienten eines Rechtsanwalts. Max Weber definiert die Soziologie als Wissenschaft vom sozialen Handeln (Weber 1922). Er gibt ein Beispiel für eine Handlung an, die nicht unter die Kategorie ,,soziales Handeln“ fällt: ,Wenn auf der Straße eine Menge Menschen beim Beginn eines Regens gleichzeitig den Regenschirm aufspannen, so ist (normalerweise) das Handeln des einen nicht an dem des andern orientiert, sondern das Handeln aller gleichartig an dem Bedürfnis nach Schutz gegen die Nässe.“ Dagegen wäre es „soziales Handeln“, wenn eine Person den Regenschirm aufspannt, weil dies die anderen tun und er nicht als Sonderling erscheinen möchte. Soziales Handeln erfordert nach Webers Definition, dass dem Handeln ein ,subjektiver Sinn“ zugeschrieben werden kann und das Handeln dem ,gemeinten Sinn 
nach auf das Verhalten anderer bezogen wird und daran in seinem Ablauf orientiert ist" (Weber 1922). Das Verhalten gegenüber dem Arzt, dem Angestellten der Autowerkstätte, dem Anlageberater oder dem Rechtsanwalt ist in diesem Sinne soziales Handeln. Allerdings haben nicht beide Vertrauensprobleme strategischen Charakter. Das Vertrauen in die Expertise des Arztes ist eine nicht-strategische, sogenannte parametrische Entscheidung (Braun und Gautschi 2011). Dagegen ist das Vertrauen des Patienten, dass die Therapieempfehlung des Arztes im Falle eines Interessenkonflikts zugunsten des Patienten ausfallen wird, strategischer Natur. Ein misstrauischer Patient kann das erste Vertrauensproblem lösen, indem er einen als kompetent empfohlenen Arzt aufsucht. Das zweite Vertrauensproblem kann er lösen, indem er einen weiteren kompetenten Arzt aufsucht, der nicht als Therapeut tätig werden und nur eine Zweitdiagnose erstellen soll, sodass kein Interessenkonflikt besteht. Das zweite Vertrauensproblem ist strategisch, weil die Ergebnisse der Entscheidungen von Patient und Arzt, von Ego und Alter, jeweils wechselseitig von der Entscheidung des anderen Akteurs abhängig sind.

Allgemein verstehen wir unter dem „sozialen Kontext“ eines Akteurs die menschengemachte Umwelt (Infrastruktur, Institutionen, soziale Normen und kulturelle Überzeugungen) und die sozialen Netzwerke und Aktivitäten, die die sozialen Handlungen eines Akteurs beeinflussen können.

Soziales Handeln im Sinne Webers kann strategisch oder parametrisch sein. Anders formuliert ist strategisches Handeln eine Teilmenge sozialen Handelns. Der andere oder die anderen strategisch handelnden Akteure bilden den sozialen Kontext, in den die Handlung eines Akteurs eingebettet ist.

Mit diesem ,strategischen sozialen Kontext“ und seiner Bedeutung für Erklärungen in der Soziologie werden wir uns im Folgenden hauptsächlich befassen. Hintergrund ist, dass viele soziologische Probleme strategischen Charakter haben, dieser aber in soziologischen Analysen häufig ausgeblendet wird. Ein Beispiel ist das instruktive Buch von Peter Hedström über „Die Anatomie des Sozialen“ (Hedström 2007). Der strategische Kontext wurde in der analytischen Soziologie allerdings vernachlässigt (Diekmann 2010).

Im Folgenden werden in Abschn. 2 zunächst die Zusammenhänge zwischen Kontext, Handlung und Effekten auf der Makroebene erläutert. Der Abschn. 3 befasst sich anhand der Hypothese der Diffusion von Verantwortung, des Wettbewerbsmodells von Raymond Boudon und des Vertrauensspiels mit der Erklärung sozialen Handelns im strategischen Kontext. Es wird gezeigt, dass strategischer sozialer Kontext die Anwendung spieltheoretischer Methoden erfordert. Wenn Handlungen strategisch sind, können die unabhängigen Variablen der Handlungstheorie nicht mehr als exogen betrachtet werden. Zudem lösen spieltheoretische Methoden das Aggregationsproblem. Ferner richtet sich das Interesse auch auf die Entwicklung von Institutionen als Reaktion auf Probleme strategischen Handelns. Institutionen können Probleme strategischen Handelns entschärfen. Sie können aber auch neue strategische Probleme erst hervorrufen. In Abschn. 4 diskutieren wir abschließend das Problem rationalen Handelns im Kontext strategisch handelnder Akteure. 


\section{Kontext, Handlung, Aggregation und Makroeffekt}

Um Kontexteffekte genauer erklären zu können, muss zunächst der Zusammenhang mit der Theorie sozialer Handlungen dargestellt werden. Sozialer Kontext beeinflusst die handlungsrelevanten Merkmale der Akteure. Einem einfachen heuristischen Schema zufolge, nehmen Handlungs- oder Entscheidungstheorien auf drei Kategorien unabhängiger Variablen Bezug. 1. Der Wert der Handlungsfolgen, 2. die wahrgenommene Wahrscheinlichkeit des Auftretens einzelner Handlungskonsequenzen und 3. die Handlungsressourcen. In der Formulierung von Hedström (2007) sind dies 1. „desires“, 2. „beliefs“, 3. „opportunities“ (DBO), bei Gintis (2007) „,beliefs, preferences, constraints“ (BPC). Präferenzen entsprechen hier den „desires“ und „constraints“ (Restriktionen) entsprechen den Opportunitäten oder Ressourcen. Opportunitäten oder Ressourcen sind z. B. Zeit, Einkommen, technologische Möglichkeiten usw. Wer einen Lottoschein am Kiosk abgibt, hat den Wunsch (die Präferenz), einen möglichst hohen Gewinn zu erzielen, den „belief“, dass eine bestimmte Wahrscheinlichkeit besteht, dass der Wunsch erfüllt wird (nicht notwendigerweise die objektive Wahrscheinlichkeit der Ziehung) und er hat einen entsprechenden Teil seines Einkommens zurückgelegt, um die Wette zu bezahlen. Die drei Elemente DBO (oder BPC) bilden noch keine vollständige Theorie. Dazu wären weiter a) Verfahren zur Messung von $\mathrm{D}$, $\mathrm{B}$ und $\mathrm{O}$ für alle Handlungsalternativen und Handlungsfolgen erforderlich sowie b) eine Entscheidungsregel, die für jede Kombination von D, B und $\mathrm{O}$ angibt, welche Handlung ausgeführt wird oder mit welcher Wahrscheinlichkeit die Handlung ausgeführt wird. Abhängig u. a. von der Erfüllung mehr oder weniger restriktiver Annahmen gibt es mehrere Entscheidungs- oder Handlungstheorien (z. B. Braun und Gautschi 2011; Eisenführ et al. 2010). Die vielzitierte Wert-Erwartungstheorie oder SEU-Theorie ist nur eine Variante von mehreren. Streng genommen ist sie auf Entscheidungsprobleme nur anwendbar, wenn auch die Annahmen (Axiome) der Theorie erfüllt sind.

Entsprechend der zugrunde gelegten Theorie beeinflusst der soziale Kontext via DBO die Handlungsergebnisse, die die Akteure mit ihren Entscheidungen hervorbringen. Die aggregierten Handlungsergebnisse entsprechen dem Effekt auf der Makroebene. Coleman (1990) hat diese Zusammenhänge in einem didaktisch vereinfachten Schema dargestellt, das allerdings die Dynamik der Prozesse ausblendet (siehe auch Esser 1999).

Betrachten wir ein Beispiel. Emile Durkheim (1983) berichtet über die Analyse statistischen Materials zur Häufigkeit von Selbstmord in unterschiedlichen sozialen Gruppierungen. So zeigt sich, dass Katholiken eine geringere Selbstmordrate aufweisen als Protestanten. Bei diesem Befund bleibt Durkheim aber nicht stehen. Er versucht, die statistische Regelmäßigkeit durch einen allgemeineren Zusammenhang zu erklären, bei dem sozialer Kontext eine Rolle spielt. Der Schlüsselbegriff ist soziale Integration. Je integrierter eine soziale Gruppe ist (messbar etwa durch die Dichte von Netzwerkbeziehungen, wechselseitige Kontakte, Häufigkeit von Kirchenbesuchen usw.), desto geringer ist die Wahrscheinlichkeit eines Selbstmords. Wenn katholische Gemeinschaften in stärkerem Maße als protestantische Gemeinschaften sozial integriert sind, lässt sich so die statistische Regelmäßigkeit erklären. Der Grad der Integration ist ein Kontextmerkmal, das die Ressourcen und Handlungsziele der 
Akteure beeinflusst. So wird es in sozial integrierten Gruppen seltener isolierte und vereinsamte Personen geben, was wiederum die Neigung zum ,,egoistischen“ Selbstmord verringert.

Bei der Analyse von Kontextmerkmalen auf das Verhalten begegnet man dem interessanten Fall, dass das gleiche Merkmal als Individual- und als Kontextmerkmal unterschiedliche Effekte auf das Verhalten ausüben kann. So könnte man vermuten, dass sich katholische Wähler in katholischen Wahlbezirken anders verhalten als in mehrheitlich protestantischer Umgebung. Der Kontext, so die Annahme, moderiert die Wahrscheinlichkeit der Wahl einer sozialdemokratischen oder konservativen Partei. Ähnlich könnte soziale Integration sowohl als Kontextmerkmal (eine Person lebt in einer mehr oder minder sozial integrierten Gruppe) und als Individualmerkmal (eine Person ist in einem bestimmten Ausmaß sozial integriert) einen Effekt auf die Wahrscheinlichkeit eines Selbstmords ausüben. Während Durkheim, in der Sprache der Statistik, den „Haupteffekt“ des Kontexts in den Mittelpunkt rückt, könnten zusätzlich das Individualmerkmal und der Interaktionseffekt (von Kontext und Individualmerkmal) eine Rolle spielen. Obwohl auch in diesem Fall das Aggregationsverfahren (im Unterschied zum strategischen Kontext) einfach ist und nur durch Summierung oder Bildung von Mittelwerten erfolgt, kann sich dennoch die Form des Zusammenhangs durch Aggregation qualitativ verändern.

Bezeichnen wir mit $Y_{\mathrm{ij}}$ die Selbstmordneigung einer Person $\mathrm{i}$ in der sozialen Gruppe $\mathrm{j}$, mit $\mathrm{X}_{\mathrm{ij}}$ das Ausmaß sozialer Integration der Person $\mathrm{i}$ in der Gruppe $\mathrm{j}$ (Individualmerkmal) und mit $X_{m j}=\left(1 / n_{j}\right) \Sigma X_{i j}$ den Mittelwert sozialer Integration in der sozialen Gruppe j (Kontextmerkmal). Bei den sozialen Gruppen kann es sich um Nachbarschaften, Wahlbezirke oder Gemeinden handeln. Dann können wir (vereinfacht) die Hypothese in eine lineare Gleichung mit einem zusätzlichen Interaktionseffekt übersetzen und die Parameter z. B. per Regressionsverfahren schätzen: $Y_{i j}=d+a X_{i j}+b$ $\mathrm{X}_{\mathrm{mj}}+\mathrm{c} \mathrm{X}_{\mathrm{ij}} \mathrm{X}_{\mathrm{mj}}$. Der Interaktionseffekt berücksichtigt den moderierenden Einfluss des Kontexts auf den Effekt des Individualmerkmals. Zur Ermittlung des Effekts auf der Makroebene kann im vorliegenden Fall durch Mittelwertbildung über die Individuen $\mathrm{i}$ in Gruppe $\mathrm{j}$ auf einfache Weise aggregiert werden. Dann erhält man die Gleichung $\mathrm{Y}_{\mathrm{mj}}=\mathrm{d}+(\mathrm{a}+\mathrm{b}) \mathrm{X}_{\mathrm{mj}}+\mathrm{cX}^{2}{ }_{\mathrm{mj}}$. Bemerkenswert ist, dass aus einer linearen Einflussbeziehung auf Individualebene (mit „,gemischtem“ Interaktionseffekt) durch Aggregation eine quadratische Gleichung auf der Kontext- oder Gruppenebene resultiert.

Bei Durkheims Analyse des Selbstmords geht es im Sinne Max Webers um „,soziales Handeln“. Die Handlungen sind aber nicht strategisch. Die Aggregation ist einfach, da sich die Selbstmordrate in einer sozialen Gruppe als Anzahl der Selbstmorde pro Jahr, dividiert durch den Umfang der Bevölkerung, ergibt.

Anders verhält es sich, wenn Handlungen strategisch miteinander verknüpft sind. Das Aggregationsproblem ist dann nicht mehr trivial und kann wesentlich komplexere Gestalt annehmen. In alltäglichen Interaktionen, in der Politik und im Wirtschaftsleben sind die Handlungen der einzelnen Akteure oft wechselseitig voneinander abhängig. Die Ressourcen (Opportunitäten im DBO-Schema) sind dann ebenso wie die wahrgenommenen Wahrscheinlichkeiten der Handlungskonsequenzen (die „,beliefs“) nicht mehr exogen gegeben, sondern endogen abhängig von den Handlungen anderer. 


\section{Die Handlungen anderer als strategischer Kontext}

\subsection{Aggregationsproblem und Makroeffekt: Das Beispiel der „Diffusion von Verantwortung“"}

Strategisches Handeln heißt, dass die Ergebnisse einer Handlung von den Handlungen anderer Akteure abhängig sind. Betrachten wir die von Darley und Latané (1968) im Experiment untersuchte Situation, dass eine Person eine Hilfeleistung benötigt. Mehrere andere Personen wissen, dass die Person Hilfe benötigt. Sie wissen auch, dass eine einzelne Person genügt, um die Hilfe zu leisten und dass andere Personen die Situation in gleicher Weise wahrnehmen. Jede Person ist auch keineswegs völlig gleichgültig und durchaus interessiert, dass Hilfe geleistet wird. Jedoch zieht es jeder Beobachter vor, dass möglichst jemand anders die Hilfeleistung erbringt. Bekanntlich kommt es nach Darley und Latané (1968) dann zu einem Prozess der „Diffusion von Verantwortung“. Je mehr Beobachter anwesend sind, desto geringer ist die Wahrscheinlichkeit, dass eine einzelne Person eingreift. Situationen der Diffusion von Verantwortung finden wir nicht nur bei Notfällen, sondern auch in vielen weniger ernsten alltäglichen Situationen ebenso wie im Wirtschaftsleben. Wenn ein Seminar von mehreren Lehrkräften durchgeführt wird, sind die einzelnen Beteiligten oftmals schlechter vorbereitet als bei alleiniger Verantwortung. Firmen warten darauf, dass andere eine Innovation entwickeln, die sie dann später kostengünstig kopieren können (Eger et al. 1992). Als allgemeines Modell für eine solche Situation ist das „Volunteer's Dilemma“ (Freiwilligendilemma) vorgeschlagen worden (Diekmann 1985).

Die Situation hat eine sehr einfache Struktur. Alle Akteure sind daran interessiert, dass das Kollektivgut (Hilfeleistung, guter Unterricht, Innovation) hergestellt wird. Den Wert des Kollektivguts bezeichnen wir mit U, die Kosten der Herstellung mit K, wobei gilt: $U>K>0$. Ein kooperativer Akteur erzielt in jedem Fall $U-K$. Ein nichtkooperativer Akteur erhält $\mathrm{U}$, aber nur wenn mindestens ein anderer Akteur kooperiert, andernfalls gehen alle leer aus. Es gibt in dieser Situation eine symmetrische, rationale „Lösung“, die mit den Mitteln klassischer Spieltheorie abgeleitet werden kann. Im sogenannten Nash-Gleichgewicht hat kein Akteur einen Anreiz, einseitig von seiner Handlungsstrategie abzuweichen, sofern die anderen Akteure die Gleichgewichtsstrategie beibehalten.

Ist $\mathrm{p}$ die Wahrscheinlichkeit der Kooperation und $\mathrm{N}$ die Anzahl der Akteure erhält man die Gleichgewichtsstrategie p* (Diekmann 1985):

$$
\mathrm{p}^{*}=1-\sqrt[N-1]{K / U}
$$

Die individuelle Kooperationsneigung verringert sich, wie erwartet, mit den Kosten und wächst mit dem Wert des Kollektivguts. Genauer gesagt, und nicht ganz selbstverständlich, sinkt die Wahrscheinlichkeit mit dem Quotienten aus den Kosten der kooperativen Handlung und dem Wert des Kollektivguts. Weiterhin sinkt die Kooperationsneigung mit der Anzahl der Akteure N. Der in Experimenten beobachtbare Effekt der Diffusion von Verantwortung lässt sich aus dem einfachen Modell ableiten. Das vorliegende Modell einer interdependenten Handlungsstruktur kann in 
verschiedene Richtungen erweitert werden. Beispiele sind das asymmetrische Freiwilligendilemma (Diekmann 1993), das Freiwilligendilemma mit Kostenteilung (Weesie und Franzen 1998) und weitere Varianten. Diesen Varianten wollen wir hier nicht nachgehen, sondern uns weiter mit der Struktur der Erklärung befassen.

Die Handlungsstruktur ist gegeben durch den Typ des Spiels und die Größen N, K und U. Sie definieren den Kontext der strategischen Situation. U kann, bezogen auf das Hilfeleistungsbeispiel, interkulturell variieren. In einer egoistischen Gesellschaft wird der Wert des Kollektivguts geringer ausfallen als in einer solidarischen Gesellschaft.

Das Nash-Gleichgewicht ist ein zentrales Element zur Lösung des Aggregationsproblems bei interdependenten Handlungen. Prognostiziert man, dass die Akteure die Gleichgewichtsstrategie anwenden, errechnet sich in der symmetrischen Situation für jede Person die gleiche, individuelle Kooperationswahrscheinlichkeit. Um den Makroeffekt abzuleiten, müssen aber noch die individuellen Wahrscheinlichkeiten aggregiert werden. „Makro“ bedeutet hier nur, dass der Effekt auf kollektiver oder Gruppenebene aus den individuellen Handlungen aggregiert wird. Im vorliegenden Fall ist der Makroeffekt der Handlungsstruktur die Wahrscheinlichkeit P, dass das Kollektivgut hergestellt wird. Die Anzahl der kooperativen Akteure folgt einer Binomialverteilung. Wenn wir nur wissen wollen, ob das Kollektivgut hergestellt wird, genügt es, die Wahrscheinlichkeit dafür zu berechnen, dass mindestens ein Akteur kooperiert. Dies ist eins minus der Wahrscheinlichkeit, dass niemand kooperiert oder $\mathrm{P}=1-\left(1-\mathrm{p}^{*}\right)^{\mathrm{N}}=1-(\mathrm{K} / \mathrm{U})^{(\mathrm{N} /(\mathrm{N}-1))}$ gemäß der oben angegebenen Gleichgewichtslösung.

Durch die Diffusion von Verantwortung nimmt die Wahrscheinlichkeit einer individuellen, kooperativen Handlung mit der Zahl der Akteure ab. Gleichzeitig sorgt aber die wachsende Zahl dafür, dass die Wahrscheinlichkeit, dass mindestens eine Person kooperiert, zunimmt. Ob letzterer Trend ersteren kompensiert, hängt vom Verlauf der Funktion ab. Aus dem Modell folgt, dass auch die Wahrscheinlichkeit P der Herstellung des Kollektivguts mit der Gruppengröße N sinkt. Empirisch wurde in Experimenten zwar festgestellt, dass sich auf der individuellen Ebene die Wahrscheinlichkeit kooperativen Handelns vermindert (Diffusion von Verantwortung), das Ausmaß der Kooperation aber über der vom Modell prognostizierten Kooperationsrate liegt (z. B. Franzen 1995). Auf der Makroebene ergibt sich dann nicht, dass die Wahrscheinlichkeit der Herstellung des Kollektivguts ebenfalls mit der Gruppengröße sinkt. Goeree und Holt (2005) sowie Tutic (2014) haben auf Basis des Freiwilligendilemmas alternative Entscheidungstheorien herangezogen, um diese Diskrepanz zu erklären ${ }^{1}$.

Zur genaueren Analyse strategischer Interaktion benötigen wir die Modelle und Lösungsbegriffe der Spieltheorie. Dies gilt besonders, wenn wir, wie in der analytischen Soziologie, an der Aufdeckung des erklärenden Mechanismus (Hedström 2007) interessiert sind. Mithilfe der Spieltheorie kann es gelingen, den sozialen

\footnotetext{
${ }^{1}$ Goeree und Holt (2005) berechnen das Gleichgewicht unter der Voraussetzung, dass die Akteure Entscheidungen mit einer gewissen Fehlerrate treffen. Für das resultierende (Quantal-Response-) Gleichgewicht können sie nachweisen, dass die Wahrscheinlichkeit, dass mindestens eine Person kooperiert, mit der Anzahl der Akteure zunimmt. Tutic (2014) erklärt den gleichen Effekt mit einem Modell begrenzter Rationalität von Rubinstein. Diese Theorien oder Modelle sind innovative Erweiterungen; ihre Validität müsste aber durch weitere empirische Tests geprüft werden.
} 
Kontext, die Handlungsstruktur, genauer zu definieren. Weiterhin folgen aus den Modellen Hypothesen über individuelle Handlungsstrategien. Gleichzeitig verhilft das Nash-Gleichgewicht zur Aggregation der individuellen Handlungen und zur Ableitung des Makroeffekts. Die drei Schritte: Kontext - individuelle Handlung aggregierter Makroeffekt können auf diese Weise präzise formuliert, die Ergebnisse an Daten geprüft werden.

\subsection{Sozialer Kontext und Opportunitäten: Das Wettbewerbsmodell von Boudon}

In der Untersuchung von Stouffer et al. (1965) wird die Zufriedenheit mit Beförderungen in zwei Armeeeinheiten berichtet. Paradoxerweise ist die Unzufriedenheit bei den häufiger beförderten Piloten höher als bei den Militärpolizisten, die im Vergleich seltener befördert wurden. Die Studie von Stouffer hat die Entwicklung und Diskussion über relative Deprivation und Bezugsgruppen stimuliert. Schon wesentlich früher hat Tocqueville (1969) auf ein ähnliches Paradox aufmerksam gemacht (Boudon 1979). Im „Alten Staat und die Revolution“ entwickelt er die Hypothese, dass Unzufriedenheit und Proteste eher in prosperierenden Perioden kulminieren. Boudon (1979) hat Tocquevilles These aufgegriffen und versucht, den soziologisch interessanten Zusammenhang unter der Prämisse rationalen Verhaltens mit einem spieltheoretischen Modell zu erklären.

Die Grundzüge des Modells seien kurz skizziert (vgl. auch Berger und Diekmann 2014; Raub 1984). In einer sozialen Gruppe des Umfangs N stehen $0 \leq k \leq N$ Positionen für Beförderungen zur Verfügung. Wer befördert werden will, muss investieren. Die Kosten dafür betragen C, der Gewinn aus der Beförderung ist B. Erfolgreiche Kandidaten erzielen $\alpha=B-C$, Verlierer erhalten $\gamma=\beta-C$ und Akteure, die nicht in eine Bewerbung investieren, erhalten eine Auszahlung in Höhe von $\beta$ (Abb. 1). Es gilt $\alpha>\beta>\gamma$. Die Anzahl von Investoren bezeichnen wir mit $n$.

Investieren heißt hier, generell Aufwand zu haben und Risiken einzugehen, um seine soziale Lage zu verbessern. Das Beispiel der Beförderung in einer Organisationseinheit von Stouffer et al. (1965) ist nur eine von vielen möglichen Anwendungen. Wer investiert, kann zum Zuge kommen oder auch nicht. Ob eine Person erfolgreich ist, hängt natürlich vom Verhalten der potenziellen Mitbewerber ab. Auf jeden Fall bleiben in einer Wettbewerbssituation $\mathrm{n}-\mathrm{k}$ frustrierte Kandidaten auf der Strecke.

\footnotetext{
Abb. 1 Investieren im Wettbewerbsmodell (nach Berger und Diekmann 2014; siehe auch Hedström 2007)
}

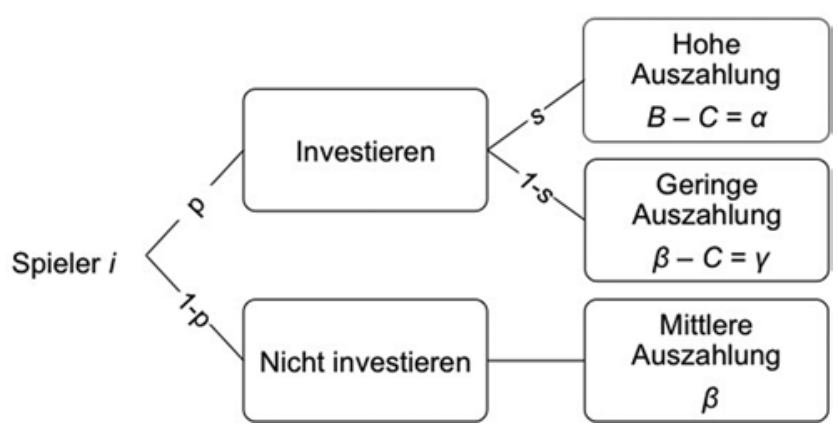


Investoren können folgende Auszahlung erwarten (Berger und Diekmann 2014):

$$
E(k, n)=\left\{\begin{array}{lr}
\frac{k}{n} \alpha+\frac{n-k}{n} y & \text { für } k<n \\
\alpha & \text { für } k \geq n .
\end{array}\right.
$$

Ein rationaler Akteur wird investieren, sofern $\mathrm{E}(\mathrm{k}, \mathrm{n})$ größer ist als $\beta$. Ist dies der Fall, unabhängig von der Zahl der Bewerber $n$, ist investieren eine dominierende Strategie. Etwas komplizierter ist die Situation, wenn ,investieren“ keine dominierende Strategie ist. In diesem Fall ist ,,investieren“ bis zu einem Schwellenwert $n^{*}$ vorteilhafter als ,nicht-investieren“. Ist die Zahl der Konkurrenten allerdings größer als der Schwellenwert, wäre es besser, auf eine Bewerbung zu verzichten. Nur wissen die Akteure natürlich nicht, wie viele Mitbewerber jeweils investieren werden. Es gibt dann mehrere Gleichgewichte in ,reinen“ Strategien, die ohne Koordination nicht erreichbar sind, sowie ein Gleichgewicht in ,gemischten“ Strategien (Berger und Diekmann 2014; Raub 1984).

Die Handlungsstruktur ist durch die Regeln des Modells und die Parameter $\alpha, \beta, \gamma$, $\mathrm{N}$ und k gegeben. Diese Elemente definieren den sozialen Kontext, in dessen Rahmen der Wettbewerb erfolgt. Die fünf Parameter sind exogen. Die Opportunitäten folgen dagegen endogen aus der Entscheidungsdynamik. Die Zahl der Konkurrenten, die die Chancen eines Kandidaten wesentlich beeinflusst, ergibt sich nämlich erst, wenn wir eine Hypothese über das Entscheidungsverhalten der Akteure annehmen. Wird nun, wie bei Boudon, die Rationalitäts-Annahme unterstellt, können verschiedene Hypothesen über den Effekt wachsender Chancen auf den Grad der Zufriedenheit oder Frustration abgeleitet werden. Unter bestimmten Bedingungen zeigt sich, dass die Zahl der Verlierer mit wachsenden Chancen (k) steigt und dann wieder sinkt. Gibt es wenige Chancen, werden nur wenige Akteure investieren. Entsprechend gibt es nur wenige Verlierer. Gibt es sehr viele Chancen, werden viele investieren. Es gibt dann viele Gewinner und ebenfalls wenige Verlierer. Problematisch ist die Situation zwischen den Extremen. Es gibt Gewinner, aber auch viele Verlierer. Der Verlauf der Zufriedenheit in Abhängigkeit von den Chancen ist u-förmig. Die Theorie oder das Modell gibt darüber Auskunft, unter welchen Bedingungen der abfallende Verlauf der Zufriedenheit, der, ,Tocqueville-Effekt", zu erwarten ist. Die aus der Theorie ableitbaren Hypothesen können sodann empirisch überprüft werden (dazu Berger und Diekmann 2014).

Der soziale Kontext definiert die Handlungsstruktur, die wiederum die Wechselwirkung zwischen Handlungsdynamik und Opportunitäten auslöst. Weil der soziale Kontext strategisches Verhalten der Akteure bewirkt, sind einfache parametrische Modelle zur Analyse der Handlungsstruktur ungeeignet. Die Opportunitäten (O im DBO-Schema) werden endogen geschaffen. Mithilfe spieltheoretischer Lösungsansätze kann die Handlungsdynamik dagegen angemessen untersucht werden. Die Nash-Gleichgewichtsstrategie ermöglicht zudem die Aggregation der Handlungsresultate. Unter bestimmten, angebbaren Bedingungen, folgt die Makrohypothese über den u-förmigen Verlauf der Zufriedenheit. 


\subsection{Sozialer Kontext und „Beliefs“: Signale des Vertrauens}

Sozialer und ökonomischer Tausch ist in der Regel mit einer doppelten Unsicherheit verbunden. Der Tausch ist zum einen zeitverzögert, d. h. einer der Tauschpartner tritt in Vorleistung. Zum andern ist nicht immer sofort ersichtlich, ob die getauschten Leistungen den Erwartungen entsprechen. Bei einem Tauschgeschäft z. B. kann es passieren, dass die gelieferte Ware von minderer Qualität ist. Es tritt ein Vertrauensproblem auf, das mit einem einfachen strategischen Modell, dem Vertrauensspiel, beschrieben werden kann.

Im Vertrauensspiel (Dasgupta 1988) interagieren zwei Akteure, der Treugeber und der Treuhänder. Der Treugeber hat die Entscheidung zwischen Kooperation (C) oder „,nicht kooperieren“ (Defektion D). Wählt er D, kommt kein Geschäft zustande. Keiner gewinnt und keiner verliert, die Auszahlung an Treugeber und Treuhänder ist jeweils null oder allgemein P. Kooperiert er dagegen, legt er sein Schicksal in die Hände des Treuhänders. Der Treuhänder wiederum kann kooperieren (z. B. die vorausbezahlte Ware liefern) oder defektieren, d. h. den Treugeber ausbeuten. Mit den üblichen Symbolen des Vertrauensspiels erhalten beide Akteure nach Kooperation den Gewinn R. Defektiert hingegen der Treuhänder, erhält er den Ausbeutungsgewinn T, der Treugeber macht einen Verlust S. Es gilt die Rangfolge der Auszahlungen $\mathrm{T}>\mathrm{R}>\mathrm{P}>\mathrm{S}$.

Beim einmaligen Vertrauensspiel wird ein rationaler Treuhänder, sobald er am Zuge ist, defektieren. Der Treugeber antizipiert dies und wird kein Vertrauen geben. Das Nash-Gleichgewicht in dieser Situation ist wechselseitige Defektion (mit Ergebnis P, P). Ein Geschäft kommt nicht zustande und beide verlieren im Vergleich zum Ergebnis wechselseitiger Kooperation, bei der sie jeweils R (Ergebnis R, R) erhalten hätten.

Tauschgeschäfte in kleinen Gemeinschaften sind nicht einmalig und nicht anonym. Oft werden wiederholte Tauschakte vorgenommen. Unter dieser Bedingung wird sich Kooperation entwickeln, wenn die zukünftig zu erwartenden Erträge aus der Tauschbeziehung hoch genug sind. Weiterhin kann es der Fall sein, dass der Treuhänder ethischen Normen folgt oder eine Reputation als ehrlicher Kaufmann hat, die er nicht aufs Spiel setzen möchte. Das Problem besteht dann aber darin, dass bei einmaligen Transaktionen oder am Anfang einer Serie von Transaktionen der Treugeber nicht weiß, ob sein Geschäftspartner vertrauenswürdig ist oder nicht.

Diese Überlegungen können mit einer Erweiterung des Vertrauensspiels präzisiert werden (Voss 1998). Wir gehen davon aus, dass es ehrliche und unehrliche Treuhänder gibt. Die ehrlichen Treuhänder sind an künftigen Geschäften interessiert mit Auszahlung $\mathrm{R}^{*}>\mathrm{T}$, für die unehrlichen gilt wie vorher $\mathrm{T}>\mathrm{R}$. Die Akteure sind in diesem Fall aufgrund ihrer Interessenlage „ehrlich“ oder ,unehrlich“. Treugeber wissen, dass es einen Anteil von $\alpha$ ehrlichen und $(1-\alpha)$ betrügerischen Geschäftspartnern gibt. Wenn sie keine weiteren Informationen haben, werden sie immer kooperieren, sofern $\alpha$ größer ist als der Schwellenwert $\alpha^{*}$, bei dem sie durch Kooperation mindestens so viel erzielen wie bei Verzicht auf Geschäftsbeziehungen, also mindestens P. Treugeber kooperieren immer, wenn $\alpha \geq \alpha^{*}=(\mathrm{P}-\mathrm{S}) /(\mathrm{R}-\mathrm{S})$ und nehmen die Verluste durch betrügerische Partner in Kauf. 
Wenn aber der Anteil betrügerischer Akteure zu groß wird $\left(\alpha<\alpha^{*}\right)$, lohnt sich Kooperation nicht mehr. In diesem Fall würden keine Geschäfte zustande kommen. Der Markt kollabiert und sämtliche Akteure, einschließlich die betrügerischen Händler, haben das Nachsehen. In solchen Situationen ist es sehr wahrscheinlich, dass Signale entstehen, mit denen Treuhänder dem Treugeber kommunizieren, dass sie ehrlich sind (dazu genauer Przepiorka und Diekmann 2013). Die betrügerischen Treugeber sind natürlich auch daran interessiert, ehrlich $\mathrm{zu}$ erscheinen. Ein Signal nutzt deshalb nur, wenn es glaubwürdig ist. Anhand des Signals muss mit hoher Wahrscheinlichkeit erkennbar sein, zu welchem „Typ“ ein Akteur gehört. Glaubwürdige Signale sind mit Kosten verbundene, beobachtbare Aktivitäten oder die beobachtbaren Ergebnisse von Aktivitäten. Genauer geht es um die Kostendifferenz bei der Produktion eines Signals. Diese Differenz muss so hoch sein, dass ehrliche Akteure trotz Signalkosten von der Kooperation profitieren $\left(\mathrm{R}^{*}-\mathrm{s}_{\mathrm{A}}>P\right)$, während betrügerische Akteure nicht gewinnen, wenn sie das Signal hervorrufen $\left(T-s_{B}<P\right)$. $\mathrm{s}_{\mathrm{A}}$ und $\mathrm{s}_{\mathrm{B}}$ bezeichnen dabei die Signalkosten der ehrlichen bzw. betrügerischen Akteure. (Die Kosten der ehrlichen, aber nicht die Kosten der unehrlichen Akteure können null sein.) Unter diesen Voraussetzungen ist ein sogenanntes separierendes Signalgleichgewicht zu erwarten. Tätowierungen waren (früher) Signale, die darüber informierten, dass eine Person im Gefängnis war und bei illegalen Geschäften mit hoher Wahrscheinlichkeit vertrauenswürdig ist (Bammann und Stöver 2006; Gambetta 2009). Polizeispitzel waren dagegen mit hoher Wahrscheinlichkeit nicht tätowiert, sofern sie ein bürgerliches Leben hatten und die (sozialen) Kosten einer Tätowierung nicht auf sich nehmen konnten. Bei der Brautwerbung existiert in den meisten Kulturen das Problem, dass es ein Kandidat mit einer dauerhaften Beziehung nicht ernst meint. Kostspielige Verlobungsgeschenke sind ein Signal, das die ehrlichen von den unehrlichen Bewerbern unterscheidet. In den USA muss ein Verlobungsring heute etwa drei Monatsgehälter kosten, berichten Uhl und Voland (2002).

Ehrliche Gebrauchtwarenhändler geben eine Garantie. Wenn sie ehrlich sind (und keine Fehler passieren), sind die Kosten null. Für unehrliche Gebrauchtwarenhändler wären dagegen die Kosten prohibitiv hoch. Das Garantieversprechen ist ein Signal, das dem Kunden erlaubt, zwischen ehrlichen und betrügerischen Händlern zu unterscheiden.

Für unsere Diskussion ist von Bedeutung, dass in Signalspielen (z. B. Diekmann 2013) die subjektiven Wahrscheinlichkeiten, dass ein Akteur zu einem bestimmten Typ gehört, erst durch die Handlungen der Akteure bestimmt werden.

Der soziale Kontext besteht aus der Spielstruktur (dem Entscheidungsbaum), den Präferenzrangfolgen der Typen von Akteuren (dem D in DBO) und den Signalkosten. Diese Elemente sind exogen gegeben. Die „beliefs“ (das B in DBO) folgen dagegen endogen aus der Handlungsdynamik. Der soziale Kontext beeinflusst die „,beliefs“ und diese wiederum die Handlungen. Im Vertrauensspiel ist dies die Entscheidung für Kooperation. Als Makroresultat ergeben sich Märkte, auf denen kooperative Tauschhandlungen vollzogen werden. 


\subsection{Institutionen}

Signale in Vertrauensbeziehungen werden dann auftreten, wenn gesetzliche oder andere institutionelle Regelungen nicht möglich oder schwer durchsetzbar sind (z. B. auf illegalen Märkten), die meisten Transaktionen einmalig sind oder selten wiederholt werden und auch keine verlässlichen Informationen über die Reputation der Akteure vorliegen. Abhängig vom jeweiligen Kontext (einmalig versus wiederholt, Verfügbarkeit verlässlicher Informationen über Reputation, funktionierendes Rechtssystem) können jeweils unterschiedliche Kooperationslösungen in Vertrauensbeziehungen entstehen. Über die Rolle sozialer Einbettung und den empirischen Test von Hypothesen zur dyadischen, institutionellen und Netzwerkeinbettung bei Vertrauensproblemen informieren Raub und Buskens (2006). DiMaggio und Louch (1998) zeigen, dass soziale Netzwerke insbesondere bei Märkten mit Vertrauensproblemen wie dem Gebrauchtwagenmarkt einen Einfluss auf die Auswahl des Händlers ausüben.

Eine Studie von Siamwalla (1978) über die Marktstruktur agrarischer Produkte in Thailand gibt Aufschluss, dass die Lösung von Vertrauensproblemen bei Transaktionen wesentlich von den Eigenschaften der gehandelten Produkte abhängen. Reis z. B. wird von den Bauern an verschiedene Zwischenhändler verkauft. Die Bindung an einen einzelnen Aufkäufer ist hier gering. Die Qualität der Ware ist vor dem Kauf gut erkennbar, sodass kein spezifisches Vertrauensproblem bezüglich der Qualität besteht. Ganz anders verhält es sich bei Gummi. Beim Kauf des Rohprodukts kann der Händler die Qualität nicht einschätzen, während der Gummifarmer selbst sehr viel besser weiß, ob das Produkt hoch- oder geringwertig ist. Die Qualität des Rohstoffs hängt nämlich von der Sorgfalt des Farmers ab, mit der unreine Stellen beseitigt wurden sowie von der Qualität der verwendeten Säure (Siamwalla 1978). Erst sehr viel später, nach der weiteren Verarbeitung, stellt sich die Qualität des Rohprodukts heraus. Aufgrund der asymmetrischen Information besteht ein Vertrauensproblem. Im Unterschied zum Reismarkt, entstand deshalb beim Gummi eine andere Marktstruktur. Die Loyalität zwischen Verkäufer und Käufern ist hoch; ein Gummifarmer hat nur wenige Aufkäufer, die diesen Farmer kennen und der Qualität der Ware vertrauen. Hinzu kommt die „Kettenstruktur“ des Handels. Ein Aufkäufer im Dorf verkauft an einen Händler im Distrikt und dieser wieder an einen Großhändler (Siamwalla 1978).

Die Eigenschaften der Ware, Gummi versus Reis, rufen in unterschiedlichem Maße Vertrauensprobleme hervor. Diese Probleme werden durch verschiedene Marktstrukturen oder Institutionen gelöst. Reismärkte sind oft Auktionsmärkte, während auf Märkten für das Rohprodukt Gummi langfristige Bindungen zwischen den Handelspartnern und Reputation charakteristisch sind (Kollock 1994). In einer experimentellen Studie hat Kollock (1994) die Märkte für Reis und Gummi simuliert. Versuchspersonen konnten unter den verschiedenen Marktbedingungen handeln, wobei sich im Ergebnis ähnliche Marktstrukturen entwickelten, wie sie für den Reisund Gummihandel berichtet wurden. Der Kontext der Akteure, und hier insbesondere Unsicherheit der Information, beeinflusst die Handlungen der Transaktionspartner und führt auf der Makroebene zu spezifischen Marktstrukturen und Institutionen.

Im digitalen Handel entstehen Vertrauensprobleme durch die Anonymität der Marktteilnehmer, die oft über große Entfernungen und Landesgrenzen hinweg Waren per Mausklick ersteigern. Die meisten Transaktionen sind einmalig; nur ein relativ 
geringer Anteil von Geschäftsabschlüssen auf den bekannten Plattformen wie eBay wird wiederholt unter den gleichen Handelspartnern getätigt. Gemäß dem StandardVertrauensspiel sollten diese Tauschakte im Nash-Gleichgewicht gar nicht stattfinden; die anonymen Märkte kollabierten. Aber es handelt sich eben nicht um ein einfaches, einmaliges Vertrauensspiel. Mit der Einführung der Möglichkeit, den Verkäufer bewerten zu können, wurde das Vertrauensproblem wesentlich entschärft. Statt des „Schattens der Zukunft“ im wiederholten Spiel wurde mit der Einführung von Reputation quasi ein „Schatten der Vergangenheit“" geschaffen. Verkäufer sind im Eigeninteresse bemüht, eine Reputation aufzubauen; Käufer wiederum honorieren die Reputation. Allerdings ist es nicht ganz selbstverständlich, dass das Bewertungssystem funktioniert, denn eigennützige Akteure würden sich die Mühe der Bewertung einfach ersparen. Der homo oeconomicus gibt kein Feedback, das Bewertungssystem würde zusammenbrechen und damit auch der Auktionsmarkt. Ein kleiner Schuss Altruismus und Reziprozität, der über den Eigennutz des homo oeconomicus hinausgeht, ist das Schmieröl anonymer, elektronischer Märkte. Das Reputationssystem ist ein einfacher, aber äußerst wirksamer institutioneller Mechanismus, der diese Milliarden schweren Märkte erst ermöglicht (Diekmann et al. 2014). Allerdings hat das wechselseitige Bewertungssystem von eBay das Ausmaß positiver Bewertungen inflationiert. Eine neue institutionelle Regelung, die ein Problem entschärft, schafft oft ein neues, das im vorliegenden Fall aber mit einer Änderung des Systems behoben werden konnte ${ }^{2}$.

Es gibt unterschiedliche institutionelle Regelungen zur Lösung von Vertrauensproblemen auf Märkten, wobei wir hier unter Institutionen dauerhafte, berechenbare Anreizmechanismen verstehen (z. B. Homann und Suchanek 2005). Neben rechtlichen Regelungen sind dies private Ordnungsmechanismen, die auf den Prinzipien von a) Loyalität (wiederholte Transaktionen unter den gleichen Partnern), b) Reputation (Bewertungen vergangener Transaktionen durch andere Handelspartner oder Bewertungen durch spezialisierte Agenturen) und c) Pfand (Hinterlegung einer Kaution) beruhen.

Die kostenlose Verfügbarkeit der Transaktionsgeschichte eines Verkäufers binnen Sekunden an jedem Ort der Welt, die Implementierung eines Reputationssystems auf Online-Plattformen, wurde erst durch die Entwicklung der Internet-Technologie ermöglicht. Andere, ,alte“ Institutionen wie „Pfänder“ (dazu Raub und Buskens 2006) lösen Vertrauensprobleme unter der Bedingung geringer Transaktionskosten. Das Pfand muss nicht den Verlust des Treugebers von $\mathrm{R}-\mathrm{S}$ bei opportunistischem Verhalten des Treuhänders ausgleichen. Es kann oft viel geringer sein, denn es muss dem Treuhänder nur den Anreiz nehmen zu betrügen $(T-R)$. Deshalb genügt in der Regel ein Pfand von einem Euro, um Supermarktkunden zu veranlassen, ihren Einkaufswagen zurückzubringen. Ein Pfand ist die übliche Absicherung gegen Vertrauensprobleme auf dem Wohnungsmarkt. Würde es keine Kautionen geben, würden auf Vermietermärkten vermutlich in (noch) stärkerem Maße Vorurteile und Diskriminierung Platz greifen (Diekmann 2013).

Der strategische Kontext der handelnden Akteure schafft ein Vertrauensproblem. Institutionelle Regelungen können das Problem entschärfen. Welche Art von Rege-

\footnotetext{
${ }^{2}$ Auch nach Reform des Systems wechselseitiger Bewertung von Käufer und Verkäufer ist die Bewertungsrate bei eBay auf erstaunlich hohem Niveau geblieben. Siehe dazu Bolton et al. (2013).
} 
lung entsteht, hängt von verschiedenen Bedingungen wie Informationsasymmetrien, Transaktionskosten und technologischem Fortschritt ab. Der Kontext strategisch handelnder Akteure ist dabei der Ausgangspunkt von Erklärungen der Entwicklung von Institutionen.

\section{Rationales Handeln, „Beauty Contest“" und begrenzte Rationalität}

Kaum ein Begriff in den Sozialwissenschaften ist so stark mit Missverständnissen behaftet wie die Begriffe „Rationalität“" oder „rationales Handeln“. Wir verstehen hier unter Rationalität nicht mehr (und nicht weniger) als konsistente Entscheidungen. Konsistenz ist das Definitionsmerkmal. Es bedeutet, dass Entscheidungen in Übereinstimmung mit den Axiomen einer Rationalitäts-Theorie getroffen werden. Diese Definition wird auch von der Erwartungsnutzentheorie Neumann-Morgensterns und der Theorie des subjektiven Erwartungsnutzens (SEU) von Savage vorausgesetzt (siehe einführend Eisenführ et al. 2010). Entsprechend dieser Definition gibt es mehr oder minder restriktive Anforderungen an den Begriff, ,rationalen Handelns“, da es unterschiedliche Entscheidungstheorien mit unterschiedlichen Axiomen-Systemen gibt. ${ }^{3}$ Allerdings werden die meisten Entscheidungstheorien wohl die Gültigkeit eines Axioms voraussetzen, nämlich Transitivität von Präferenzen: Wenn A der Alternative B vorgezogen wird und B der Alternative C, dann sollte auch A der Vorzug gegenüber $\mathrm{C}$ gegeben werden.

Unsere Definition steht nicht im Gegensatz zur Definition des „frühen“ Boudon, dessen Wettbewerbsmodell weiter oben behandelt wurde. Die Definition steht allerdings im Gegensatz zu Boudons (2013) Konzept von Rationalität (oder „,kognitiver Rationalität"), das er in späteren Schriften ausgearbeitet hat (dazu auch Boudon in diesem Band). Es steht auch nicht im Einklang mit Konzepten, die spezifische Motive als Komponenten der Definition von Rationalität einfordern.

Rationalität erfordert nicht Eigennutz und schon gar nicht materiellen Eigennutz. Der homo oeconomicus handelt per Definitionem rational, aber rationales Handeln ist nicht gleichbedeutend mit dem Handeln des fiktiven homo oeconomicus.

Rationalität und Altruismus müssen keine Gegensätze sein. Menschen können altruistisch rational handeln im Sinne des Konsistenzkriteriums. Andreoni und Miller (2002) haben gezeigt, dass Akteure mit altruistischen Präferenzen rational in dem Sinne handeln können, dass sie die Axiome der Entscheidungstheorie erfüllen. Wenn Menschen nicht dem Bild des homo oeconomicus entsprechen und zudem noch altruistische Ziele verfolgen, folgt nicht zwangsläufig, dass sie irrational handeln. Im Sinne des Konsistenzkriteriums können ihre Entscheidungen strikt rational sein.

„Rationalität“ wird „motivfrei“" definiert, ohne bestimmte Präferenzen / Ziele / desires auszuschließen. Die axiomatische Nutzentheorie von Neumann-Morgenstern oder Savage (Braun und Gautschi 2011; Eisenführ und Weber 2010) hat schon immer die „weite“ Version der Rational-Choice-Theorie (Opp 1999; Finkel 2008) eingeschlossen. Allerdings muss es Hinweise auf die empirische Gültigkeit der Annahmen

\footnotetext{
${ }^{3}$ Für die deskriptive Anwendung einer Entscheidungstheorie ist es ohnehin unerheblich, ob man ein Verhalten als ,rational“ bezeichnet oder nicht.
} 
geben, die zumindest näherungsweise erfüllt sein sollten. Dieser Aspekt gerät bei Anwendungen, insbesondere in der Soziologie, allzu oft ins Hintertreffen.

Im strategischen Kontext kommt eine weitere Rationalitäts-Anforderung hinzu, die empirisch nicht immer erfüllt ist: „,common knowledge“. Damit ist gemeint, dass alle Akteure bestimmte (aber nicht notwendigerweise die gleichen) Informationen über die Spielstruktur und die Auszahlungen haben und alle wissen, dass alle dieses Wissen haben usw.

Betrachten wir eine Situation, in der genau diese Annahme kritisch ist. Das „Beauty-Contest-Spiel“" erhielt den Namen von einem Zitat von Keynes, in dem er den Kauf von Aktien mit einem Schönheitswettbewerb vergleicht (Selten und Nagel 1998). Auf Aktienmärkten geht es nicht darum, sich für die der eigenen Meinung nach beste Aktie zu entscheiden, sondern für diejenige, von der man glaubt, dass die anderen sie für die beste Entscheidung halten. Mehr noch, nämlich von der man glaubt, dass alle glauben, dass die anderen sie als besten Kauf bewerten usw. Die Regeln des Beauty-ContestSpiels sind einfach. Es gibt N Teilnehmer, die eine Zahl zwischen null und 100 wählen sollen. Einen Preis erhält, wer 2/3 des (arithmetischen) Mittelwerts aller genannten Zahlen am nächsten kommt. Bei mehreren Gewinnern wird der Preis geteilt.

Würde man nicht-strategisch überlegen, könnte man sagen: „Alle Zahlen sind gleichverteilt, der Mittelwert sollte daher bei 50 liegen. Ich wähle 33 1/3, denn das sind 2/3 von 50." Strategisches Denken auf der ersten Stufe geht einen Schritt weiter. „Wenn alle diese Überlegung anstellen, wird der Mittelwert 33 1/3 sein. Ich wähle deshalb 22 2/9.“ Auf der nächsten Stufe strategischen Denkens sind es ungefähr 15 usw., bis man sich schließlich in fortwährenden Iterationen der Null annähert.

Die Wahl von „Null“ ist auch die einzige strikte Nash-Gleichgewichtsstrategie. Das Ergebnis im Gleichgewicht ist zudem Pareto-optimal, d. h. es gibt keine andere Kombination von Strategien, bei der Akteure mehr erzielen können, ohne dass andere Nachteile erleiden. Bei einem bindenden Vertrag würden, anders als im Gefangenendilemma, alle Akteure das Nash-Gleichgewicht vereinbaren. Nach üblichen Kriterien der klassischen Spieltheorie wäre die Nash-Gleichgewichtsstrategie rational. Wenn entsprechend der verhaltensorientierten Spieltheorie „Ungleichheitsaversion“ als Motiv hinzukommt (Fehr und Schmidt 1999), würde sich daran nichts ändern. Im Gegenteil, es wäre ein weiterer Grund für die Wahl der Rationalitäts-Strategie, da diese auch noch zu maximaler Gleichheit führt.

In Experimenten wählen allerdings nur relativ wenige Akteure die GleichgewichtsStrategie. Die Wahlen häufen sich typischerweise bei rund 33 oder 22. Wenn man nun die Entscheidungssituation erneut vorgibt und über den Mittelwert informiert, wird man Werte bei 15 oder 10 erhalten und mit weiteren Runden sich dem Gleichgewicht annähern (Selten und Nagel 1998). Diesen Prozess beobachtet man auch dann, wenn genauere Informationen über die Verteilung der Vorrunde gegeben werden (Diekmann 2009). Man könnte nun annehmen, dass die Akteure die Gleichgewichtsstrategie nicht wählen, weil sie diese nicht kennen. In einem Vorlesungs-Experiment wurde daher der Hälfte der Teilnehmer die Nash-Gleichgewichtsstrategie mitgeteilt, dagegen nicht den Teilnehmern in der Kontrollgruppe. Zwar lagen nun die gewählten Zahlen in der informierten Gruppe unter den Durchschnittswerten der Kontrollgruppe, aber keineswegs wurde überwiegend die „Rationalitäts-Strategie“ gewählt. Warum handeln die Akteure ,,irrational“" gemessen am Kriterium des „,common knowledge“? Die Antwort 
lautet vermutlich: Selbst wenn man weiß, dass es rational wäre, null zu wählen, so ist doch damit zu rechnen, dass ein Teil der Mitspieler dies nicht tun wird (Selten und Nagel 1998). Dann aber ist es sinnvoller nicht die Gleichgewichtsstrategie zu wählen. Also wird man selbst auch nicht diese Strategie wählen. Die vermutete Irrationalität der Anderen hat zur Folge, dass ein Akteur sich nicht für die Gleichgewichts-Strategie entscheiden wird und somit selbst irrational handelt. Diese Entscheidung ist klug, denn in keinem der publizierten Beauty-Contest-Experimente hat jemals ein Akteur mit der Wahl der Gleichgewichtsstrategie in der ersten Runde einen Gewinn erzielt. Das Common-Knowledge-Kriterium wechselseitiger Rationalität und des Wissens darüber ist im strategischen Kontext keineswegs immer erfüllt.

Das Beauty-Contest-Spiel wurde zur Erklärung von spekulativen „Blasen“ auf Aktien- oder Immobilienmärkten herangezogen. Selbst wenn man weiß, dass es sich um eine Blase handelt und man das Platzen der Blase erwartet (aber der Zeitpunkt ungewiss ist), kann es sich lohnen zu investieren. Denn die Preise können bis zum Eintreten der Katastrophe noch weiter steigen. Ähnlich wählen Akteure im Beauty-Contest-Spiel zumindest zeitweilig die ,irrationale“ Strategie, obwohl ihnen die Gleichgewichts-Strategie bekannt ist. Plastisch formulierte der Chef der Citygroup, Charles Prince, diese Überlegung in der ,Financial Times“ im Juli 2007, kurz vor Ausbruch der Krise: ,When the music stops, in terms of liquidity, things will be complicated. But as long as the music is playing, you've got to get up and dance. We're still dancing“" (nach Akerlof and Shiller 2010, S. XII). Im folgenden Jahr platzte die Blase!

Die stufenweise Annäherung an die Gleichgewichts-Strategie mit wachsender Rundenzahl des „Beauty Contest“ demonstriert, dass Gleichgewichtspunkte im Zuge eines evolutionären Prozesses durch Lernen und adaptives Verhalten erreicht werden können. Lernen, adaptives und myopisches Verhalten beobachtet man oft in experimentellen Studien. Theorien begrenzter Rationalität (,bounded rationality“), die explizit von Prinzipen adaptiven Verhaltens ausgehen, sind in diesen Fällen strikten Rationalitäts-Theorien (als deskriptive, prognostische Theorie) überlegen.

\section{Ausblick}

Pioniere in der Soziologie wie Goffman (1969) oder Boudon (1979) haben erkannt, dass in vielen Fällen strategisches Handeln der Schlüssel zur Erklärung gesellschaftlicher Prozesse ist. Soziale Handlungen im Sinne Max Webers haben strategischen Charakter, wenn sie nicht nur an den Handlungen anderer ausgerichtet, sondern die Ergebnisse von den Handlungen anderer abhängig sind. Dies unterscheidet bloße Imitation von einer Massenpanik, einem Börsenkrach oder dem Wachstum einer Protestbewegung. Heute stehen zunehmend verfeinerte Modelle der klassischen und der verhaltensorientierten Spieltheorie zur Verfügung, mit deren Hilfe es erst möglich ist, den strategischen Kontext, die Struktur strategischer Interaktionen, präzise zu beschreiben und daraus prüfbare Folgerungen abzuleiten. Die Grundlagenprobleme soziologischer Forschung haben strategischen Charakter: Die Entstehung sozialer Ordnung, die Probleme von Kooperation, Konflikt und dem Zerfall von Ordnung, die Befolgung sozialer Normen und das Problem der Sanktionierung, die Entstehung von Institutionen, sozialer Tausch und Vertrauensproblem, die Entwicklung von Pro- 
testbewegungen, soziale Dilemmas und kollektive Güter. Sowohl in der Ausbildung als auch in der Forschung der Soziologie sollten die modernen Methoden der Analyse strategischer Interaktionen größere Verbreitung finden.

Die aus Modellen strategischen Handelns ableitbaren Hypothesen können experimentell, in Feldexperimenten, Survey-Studien oder mit anderen Methoden untersucht werden. Die experimentelle Methode hat den Vorteil, dass durch Randomisierung Kausalbeziehungen genauer nachweisbar sind. Das Laborexperiment in der Verhaltensforschung ist aber nur ein erster Filter, denn die untersuchten Hypothesen sollten sich danach auch in Feldexperimenten oder anderen Studien bewähren, um als valide gelten zu können.

Die klassische Spieltheorie erfordert starke Rationalitäts-Annahmen. In Situationen, in denen diese Modelle hinreichend valide Prognosen erlauben, besteht kein Grund, alternative Entscheidungsprinzipien heranzuziehen. Zudem liefert die Theorie einen Referenzpunkt. Weicht das beobachtete Verhalten davon ab, stellt sich die Herausforderung, die „Anomalie“ zu erklären. Auf diese Weise wurden oft neue Hypothesen und Erklärungen hervorgebracht. Wenn die strikten Rationalitäts-Modelle versagen, wie beim Beauty Contest demonstriert, kann auf Modelle „begrenzter“ Rationalität zurückgegriffen werden. Wäre hingegen die Rational-Choice-Theorie eine mechanisch anwendbare allgemeine Theorie, könnte man die Theoriekonstruktion einem Computer überlassen. Rational-Choice-Theorie, analytische Soziologie, Spieltheorie und psychologische Entscheidungsforschung sind stattdessen als eine ,Tool Box“ aufzufassen. Sie liefern heuristische Prinzipien und Instrumente, um in einem konkreten Anwendungsbereich eine „Theorie mittlerer Reichweite“ (Merton 1949) zu konstruieren.

Die skizzierten Anwendungen folgen der Makro-Mikro-Makro-Logik, wobei diese aber oft wesentlich komplizierter ist, als im vereinfachten „Coleman-Boot“" dargestellt. Strategischer sozialer Kontext beeinflusst die unabhängigen Variablen der Entscheidungstheorie. Die Präferenzen (D), die Wahrnehmung von Chancen (B) und die Ressourcen $(\mathrm{O})$ hängen vom Kontext, aber auch vom strategischen Verhalten der anderen Akteure ab. Spieltheoretische Modelle präzisieren diese Zusammenhänge, sofern die Handlungen strategisch miteinander verknüpft sind, und ermöglichen die Ableitung empirisch prüfbarer Hypothesen. Mit dem Nash-Gleichgewicht (oder dessen Verfeinerungen) liefern sie gleichzeitig eine Aggregationsregel, d. h. eine Aussage über den Effekt auf der Makroebene. Denn die Wahl einer Gleichgewichtsstrategie ist eine individuelle Entscheidung und berührt die Mikroebenene, während das aus den Handlungen aller Akteure resultierende Gleichgewicht die Makroebene betrifft. Der Begriff „Makro“ kann sich auf eine kleine Gruppe von mindestens zwei Akteuren, eine große Zahl von Teilnehmern an einem Markt wie eBay oder z. B. bei Allmendemodellen zum Klimawandel auf die Weltgesellschaft beziehen.

Diese Zusammenhänge gelten auch für Modelle aus der verhaltensorientierten (,behavioral“) Spieltheorie, die die Annahmen der klassischen Theorie durch psychologische Motive wie z. B. „Ungleichheitsaversion“ (Fehr und Schmidt 1999) ergänzen. Wie sich beim „Beauty Contest“ zeigte, sind es oft nicht rationale, vorausschauende Entscheidungen, die direkt zu einer Gleichgewichtssituation führen. Vielmehr wird das Gleichgewicht (eventuell) erst im Zuge eines evolutionären Prozesses erreicht. Gürerk et al. (2006) konnten in einem bemerkenswerten Experiment zeigen, wie sich Sanktionsinstitutionen in einer Kollektivgutsituation evolutionär herausge- 
bildet haben, obwohl die Versuchspersonen anfänglich die sanktionsfreien Institutionen bevorzugt hatten. Durch eine „Abstimmung mit den Füßen“ fand die erfolgreiche, kooperationsfördernde Institution zunehmend mehr Anhänger und setzte sich schließlich gegen die sanktionsfreie Regelung durch. Evolutionäre Spieltheorie (Young 2001) untersucht die Dynamik der Prozesse und kommt mit wesentlich schwächeren Rationalitätsannahmen aus als die klassische Rational-Choice- und Spieltheorie.

Danksagung Ich bedanke mich bei Jürgen Friedrichs und den anonymen Gutachtern für Hinweise und Anregungen.

\section{Literatur}

Akerlof, George A., und Robert J. Shiller. 2010. Animal spirits. 9. Aufl. Princeton: Princeton University Press.

Andreoni, James, und John Miller. 2002. Giving according to GARP: An experimental test of the consistency of preferences for altruism. Econometrica 70:737-753.

Bammann, Kai, und Heino Stöver. Hrsg. 2006. Tätowierungen im Strafvollzug. Hafterfahrungen, die unter die Haut gehen. Oldenburg: BIS-Verlag.

Berger, Joël, und Andreas Diekmann. 2014. The logic of relative frustration. Boudon's competition model and experimental evidence. Working paper ETH Zurich.

Bolton, Gary E., Ben Greiner und Axel Ockenfels. 2013. Engineering trust: Reciprocity in the production of reputation information. Management Science 59:265-285.

Boudon, Raymond. 1979. Widersprüche sozialen Handelns. Neuwied: Luchterhand.

Boudon, Raymond. 2013. Beiträge zur allgemeinen Theorie der Rationalität. Tübingen: Mohr-Siebeck.

Braun, Norman, und Thomas Gautschi. 2011. Rational Choice Theorie. München: Juventa.

Coleman, James. 1990. Foundations of social theory. Cambridge, MA: Harvard University Press.

Darley, John M., und Bibb Latané. 1968. Bystander intervention in emergencies. Diffusion of responsibility. Journal of Personality and Social Psychology 8:377-383.

Dasgupta, Partha. 1988. Trust as a commodity. In Trust. Making and breaking cooperative relations, Hrsg. Diego Gambetta, 49-72. Oxford: Blackwell.

Diekmann, Andreas. 1985. Volunteer's dilemma. Journal of Conflict Resolution 29:605-610.

Diekmann, Andreas. 1993. Cooperation in an asymmetric volunteer's dilemma game. Theory and experimental evidence. International Journal of Game Theory 22:75-85.

Diekmann, Andreas. 2009. Rational choice, evolution and the „Beauty Contest“. In Raymond Boudon - A Life in Sociology, Hrsg. Mohamed Cherkaoui und Peter Hamilton, 1-12. Oxford: Bardwell Press.

Diekmann, Andreas. 2010. Analytische Soziologie und Rational Choice. In Die analytische Soziologie in der Diskussion, Hrsg. Thomas Kron und Thomas Grund, 193-204. Wiesbaden: VS Verlag für Sozialwissenschaften.

Diekmann, Andreas. 2013. Spieltheorie, Einführung, Beispiele, Experimente. 3. Aufl. Reinbek: Rowohlt.

Diekmann, Andreas, Ben Jann, Wojtek Przepiorka und Stefan Wehrli. 2014. Reputation formation and the evolution of cooperation in anonymous online markets. The American Sociological Review 79:65-85.

DiMaggio, Paul, und Hugh Louch. 1998. Socially embedded consumer transactions: For what kinds of purchases do people most often use networks? American Sociological Review 63:619-637.

Durkheim, Emile. 1983. Der Selbstmord. 1897. Frankfurt a. M.: Suhrkamp.

Eger, Thomas, Manfred Kraft und Peter Weise. 1992. On the equilibrium proportion of innovation and imitation. A game-theoretic approach. Economics Letters 38:93-97.

Eisenführ, Franz, Martin Weber und Tomas Langer. 2010. Rationales Entscheiden. 5. Aufl. Berlin: Springer.

Esser, Hartmut. 1999. Soziologie. Spezielle Grundlagen. Bd. 1 Situationslogik und Handeln. Frankfurt a. M.: Campus.

Fehr, Ernst, und Klaus M. Schmidt. 1999. A theory of fairness, competition, and cooperation. The Quarterly Journal of Economics 114:817-868. 
Finkel, Steven E. 2008. In defense of the „wide“ rational choice model of collective political action. In Rational Choice. Theoretische Analysen und empirische Resultate. Festschrift für Karl-Dieter Opp, Hrsg. Andreas Diekmann, Klaus Eichner, Peter Schmidt und Thomas Voss, 23-36. Wiesbaden: VS Verlag für Sozialwissenschaften.

Franzen, Axel. 1995. Group size and one-shot collective action. Rationality and Society 7:183-200.

Gambetta, Diego. 2009. Codes of the underworld: How criminals communicate. Princeton: Princeton University Press.

Gigerenzer, Gerd. 2013. Risiko. Wie man die richtigen Entscheidungen trifft. München: Bertelsmann.

Gintis, Herbert. 2007. A framework for the unification of the behavioral sciences. Behavioral and Brain Sciences 30:1-61.

Goeree, Jacob A., und Charles A. Holt. 2005. An explanation of anomalous behavior in models of political participation. American Political Science Review 99:201-213.

Goffman, Erving. 1969. Strategic interaction. Philadelphia: University of Pennsylvania Press.

Gürerk, Özgür, Bernd Irlenbusch und Bettina Rockenbach. 2006. The competitive advantage of sanctioning institutions. Science 312:108-111.

Hedström, Peter. 2007. Dissecting the social. On the principles of analytical sociology. Cambridge: Cambridge University Press.

Homann, Karl, und Andreas Suchanek. 2005. Ökonomik. Eine Einführung. 2. Aufl. Tübingen: Mohr-Siebeck.

Kollock, Peter. 1994. The emergence of exchange structures: An experimental study of uncertainty, commitment, and trust. American Journal of Sociology 100:313-345.

Merton, Robert K. 1949. Social theory and social structure. New York: The Free Press.

Opp, Karl-Dieter. 1999. Contending conceptions of the theory of rational action. Journal of Theoretical Politics 11:171-202.

Przepiorka, Wojtek, und Andreas Diekmann. 2013. Temporal embeddedness and signals of trustworthiness: Experimental tests of a game theoretic model in the United Kingdom, Russia, and Switzerland. European Sociological Review 29:1010-1023.

Raub, Werner. 1984. Rationale Akteure, institutionelle Regelungen und Interdependenzen. Untersuchungen zu einer erklärenden Soziologie auf strukturell-individualistischer Grundlage. Frankfurt a. M.: Peter Lang.

Raub, Werner, und Vincent Buskens. 2006. Spieltheoretische Modellierungen und empirische Anwendungen in der Soziologie. In Methoden der Sozialforschung. Sonderheft 44 der Kölner Zeitschrift für Soziologie und Sozialpsychologie, Hrsg. Andreas Diekmann, 560-598. Wiesbaden: VS Verlag für Sozialwissenschaften.

Selten, Reinhard, und Rosemarie Nagel. 1998. Das Zahlenwahlspiel. Hintergründe und Ergebnisse. Spektrum der Wissenschaft 2:16-22.

Siamwalla, Ammar. 1978. Farmers and middlemen. Aspects of agricultural marketing in Thailand. Economic Bulletin for Asia and the Pacific 29:38-50.

Stouffer, Samuel A., Edward A. Suchman, Leland C. De Vinney, Shirley A. Star und Robin M. Williams Jr. 1965. The American soldier. 1949. Manhattan: Military Affairs, Aerospace Historian Publishing.

Tocqueville, Alexis de. 1969. Der alte Staat und die Revolution. 1856. Reinbek: Rowohlt.

Tutić, Andreas. 2014. Procedural rational volunteer. Journal of Mathematical Sociology 38:219-232.

Uhl, Matthias, und Eckart Voland. 2002. Angeber haben mehr vom Leben. Heidelberg: Spektrum.

Voss, Thomas. 1998. Vertrauen in modernen Gesellschaften. In Der Transformationsprozess, Hrsg. Regina Metze, Kurt Mühler und Karl-Dieter Opp, 91-129. Leipzig: Universitätsverlag.

Weber, Max. 1922. Wirtschaft und Gesellschaft. Tübingen: Mohr.

Weesie, Jeroen, und Axel Franzen. 1998. Cost sharing in a volunteer's dilemma. Journal of Conflict Resolution 42:600-618.

Young, Peyton. 2001. Individual strategy and social structure. An evolutionary theory of institutions. Princeton: Princeton University Press. 
Andreas Diekmann, 1951, Dr. rer. pol., Professor für Soziologie an der ETH Zürich. Forschungsgebiete: Theorien sozialer Kooperation, Methoden und Modelle in der Sozialforschung, Bevölkerungs- und Umweltsoziologie, experimentelle Spieltheorie. Neuere Veröffentlichungen: Reputation formation and the evolution of cooperation in anonymous online markets. American Sociological Review 79, 2014 (mit B. Jann, W. Przepiorka und S. Wehrli); Subjective discount rates in the general population and their predictive power for energy saving behavior. Energy Policy 65, 2014 (mit H. Bruderer-Enzler und R. Meyer); The intergenerational transmission of divorce: A fifteen-country study with the fertility and family survey. Comparative Sociology 12, 2013 (mit K. Schmidheiny); Individual heterogeneity and costly punishment: A volunteer's dilemma. Proceedings of the Royal Society Biological Sciences 280, 2013 (mit W. Przepiorka); Temporal embeddedness and signals of trustworthiness: Experimental tests of a game theoretic model in the United Kingdom, Russia, and Switzerland. European Sociological Review 29, 2013 (mit W. Przepiorka); Making use of «Benford's law〉 for the randomized response technique. Sociological Methods and Research 41, 2012. 